\title{
A scoping review to inform a multi-disciplinary approach for nutrition therapy in critically ill children with pressure injuries
}

\author{
Katarina G. Berry $^{1 \#}$, Stephanie M. Seiple ${ }^{1 \#}$, Judith J. Stellar ${ }^{2}$, Monica L. Nagle ${ }^{1}$, Kelsey Curry ${ }^{1}$, \\ Amanda Immel ${ }^{3}$, Richard James ${ }^{4}$, Vijay Srinivasan ${ }^{5,6}$, Maria R. Mascarenhas ${ }^{7,8}$, Anna Garrett ${ }^{3}$, \\ Sharon Y. Irving ${ }^{9,10}$
}

${ }^{1}$ Department of Clinical Nutrition, Children's Hospital of Philadelphia, Philadelphia, PA, USA; ${ }^{2}$ Department of Nursing and General Surgery, Children's Hospital of Philadelphia, Philadelphia, PA, USA; ${ }^{3}$ Department of Nursing, Children's Hospital of Philadelphia, Philadelphia, PA, USA; ${ }^{4}$ University of Pennsylvania Biomedical Library, University of Pennsylvania, Philadelphia, PA, USA; ${ }^{5}$ Department of Anesthesiology and Critical Care Medicine, Children's Hospital of Philadelphia, Philadelphia, PA, USA; ${ }^{6}$ Department of Anesthesiology and Critical Care, University of Pennsylvania School of Medicine, Philadelphia, PA, USA; ${ }^{7}$ Division of Gastroenterology, Hepatology and Nutrition, Children's Hospital of Philadelphia, Philadelphia, PA, USA; ${ }^{8}$ Professor of Pediatrics, Perelman School of Medicine, University of Pennsylvania, Philadelphia, PA, USA; ${ }^{9}$ Pediatric Nursing, University of Pennsylvania School of Nursing, Philadelphia, PA, USA; ${ }^{10}$ Department of Nursing and Clinical Care, Critical Care Children's Hospital of Philadelphia, Philadelphia, PA, USA

Contributions: (I) Conception and design: All authors; (II) Administrative support: None; (III) Provision of study materials or patients: None; (IV) Collection and assembly of data: All authors; (V) Data analysis and interpretation: None; (VI) Manuscript writing: All authors; (VII) Final approval of manuscript: All authors.

\#These authors contributed equally to this work.

Correspondence to: Katarina G. Berry, MS, RD, LDN. Department of Clinical Nutrition, Children's Hospital of Philadelphia, 3401 Civic Center Blvd., Philadelphia, PA 19104, USA. Email: berryk5@chop.edu.

\begin{abstract}
Nutrition status plays a critical role in pressure injury (PI) healing and yet the available literature, especially in pediatric patients, is limited. Critically ill pediatric patients are at an increased risk of skin integrity compromise and PI development. Adequate nutritional intake can often be challenging to achieve in this population and immobility and illness present additional obstacles to maintaining skin integrity in this vulnerable population. Despite the unique nutritional challenges and needs of this group, there is no standardized approach to macro- and micronutrient management and monitoring. Here, several key vitamins and minerals believed to play a role in PI healing are discussed and an approach to nutritional management and monitoring for PI healing in pediatric patients is proposed. Registered dietitians (RD) are essential to assess individual patient macro and micronutrient requirements, to identify gaps and make recommendations to optimize nutritional therapy that may exist and impact wound healing. We used a scoping review to focus on the interplay of nutrition and PI healing and inform a multidisciplinary approach to PI identification and management. Through this review, we propose a strategy for the nutritional management of pediatric patients $<30 \mathrm{~kg}$ at risk for and who present with PI.
\end{abstract}

Keywords: Pressure injury (PI); wound healing; nutrition management; micronutrients; macronutrients; pediatrics

Submitted Jan 05, 2021. Accepted for publication Jul 22, 2021.

doi: $10.21037 / \mathrm{tp}-21-3$

View this article at: https://dx.doi.org/10.21037/tp-21-3

\section{Introduction}

The skin is the largest organ system whose primary function is to serve as a barrier to protect the body against foreign microbes and noxious substances. Skin integrity and function require macro and micronutrients for cell turnover to maintain homeostasis, and support new growth, and repair, especially in the event of injury or decreased integrity. Immobility, illness, or injury coupled 
with risk of suboptimal nutrient intake put the skin at risk for compromise, particularly in hospitalized infants and children, a population that presents unique challenges for nutrition therapy and optimal skin care. In addition to providing for the metabolic demands of their current disease, illness or injury, nutrition therapy must also address any pre-existing malnutrition, minimize any deterioration of nutrition status and growth milestones.

A common compromise of skin barrier in hospitalized children is the occurrence of a pressure injury (PI). Defined in terms of the localization of injury to skin or underlying tissue singularly imputable to pressure or a combination of pressure and skin shear (1), the incidence of PI (expressed as the number of PI per 1,000 patient days) in children reportedly ranges between $10 \%$ to $27 \%(2,3)$. The prevalence of PI (number of patients affected / total patient population at a given point in time) in children is reported to be as high as $43 \%$ (1). In hospitalized patients, the combined prevalence and incidence of PI define its occurrence [per patient days, or per device days in the case of medical device related pressure injury (MDRPI)]. In a series of three prevalence surveys of hospital acquired skin injury (HASI), Stellar et al. (2013) report PI to be the most common type of skin injury in children (4). The etiology of these injuries includes immobility, MDRPI, diaper dermatitis and epidermal stripping. Overall, current data suggests that $1.4 \%$ of all infants and children experience a PI during hospitalization $(1,5)$.

Skin compromise can be categorized into two broad groups-those caused by intrinsic factors and those due to extrinsic causes, both of which can result in a PI. Intrinsic factors may be the underlying disease entity, including autoimmune and genetic conditions, immune compromised status, conditions requiring surgical intervention, moisture associated skin disruption and skin breakdown associated with medication or therapeutic treatments. Extrinsic factors that contribute to skin compromise include immobility, device related damage, extravasation wounds, and medical adhesive related skin injury (MARSI), also known as epidermal stripping.

Skin compromise is an inherent adverse event risk for children in the Pediatric Intensive Care Unit (PICU) where immobility, disease and/or illness and injury are common, and optimal nutrition therapy is often a challenge. Point prevalence survey studies report the incidence of PI in the PICU population to range from $9 \%$ to $28 \%(6-8)$ and as high as $23 \%$ in neonates (9). Addressing skin compromise in hospitalized children requires intentional focus and multidisciplinary care. While optimal nutrition therapy through provision of macronutrients is an important consideration for wound healing and maintenance of skin integrity, the role of micronutrient supplement therapy in PI prevention and treatment is less well defined.

Recently published international clinical practice guidelines identified suboptimal nutritional status as a significant risk factor for PIs in adults (10). Though these guidelines do not specifically address infants and children, the authors infer similar assumptions for pediatric patients. There is a dearth of information on the role of micronutrients in prevention of PI and little guidance on micronutrient supplementation therapy for PI management in hospitalized pediatric patients. In this paper, we aim to review the available literature related to PI in critically ill children, identify best practice assessment tools to identify PICU patients at risk for PI, describe macro and micronutrient requirements in children in the PICU, and discuss a multidisciplinary approach to the prevention, identification and management of PI in critically ill pediatric patients.

\section{Methods}

We performed a scoping review to characterize the importance of nutrition status and therapy (including macro and micronutrients) in the context of PI in hospitalized or critically ill children. A scoping review was employed as there is limited published literature on this topic. Scoping reviews are an increasingly important methodology to systematically explore and describe broad research topics, and to set the stage for further, more specific investigations such as systematic reviews (11). Scoping reviews differ from other review methods in that they may accommodate and include sources that are not empirical or have a rigorous research methodology. Additionally, scoping reviews do not perform critical appraisal or assessment on the selected evidence. The PubMed, Embase, and CINAHL databases were searched using keywords and index terms for elements and nutrients of interest in a nutrition-related context. This search was combined with searches for articles focused on wounds or wound healing and limited to results from the past ten years with pediatric patients as the focus or study population. The search strategy used for this review is 
Table 1 Search strategy with mesh terms

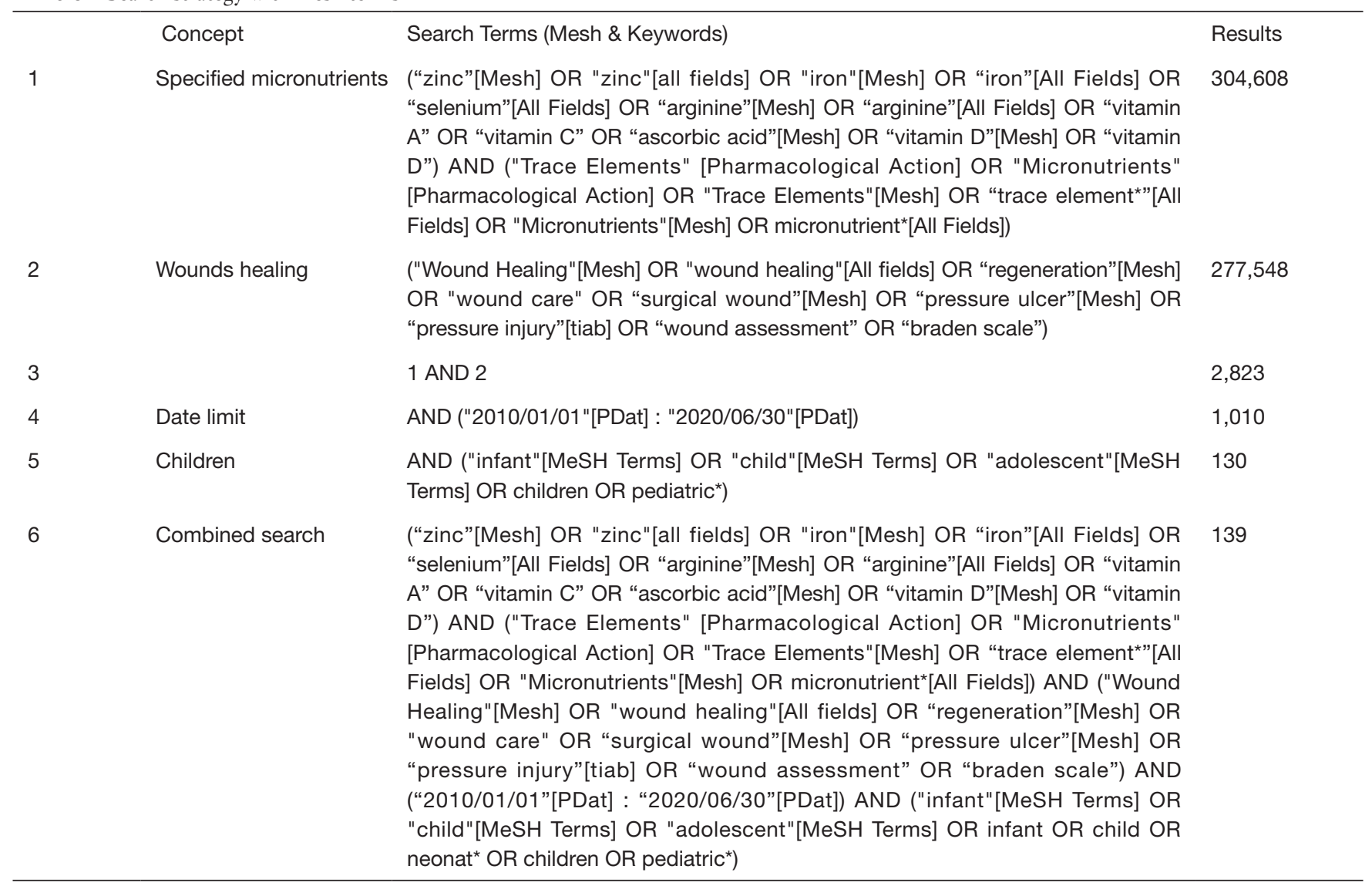

presented in Table 1.

\section{Results}

After the specified databases were searched and citations identified, additional citations were added by hand searches from published research known to the authors resulting in a combined total of 553 articles. Once duplicate articles were removed, two authors (KB, SS) then screened citations by title and abstract according to broad characteristics of language, publication type, and population $(\mathrm{n}=528)$. The retained citations were subsequently screened according to exclusion and inclusion criteria developed by the research team. Eligible citations were acquired in full text and distributed to all authors for review and synthesis. Figure 1 is the PRISMA flow diagram of the article selection process. In total, we reviewed 82 full text articles to develop this narrative, what follows is a summary of macro and micronutrients in the context of pediatric critical illness.

\section{Energy}

Estimated energy needs should account for the hypermetabolic state of the hospitalized patient as well as the need for anabolic cell regeneration to heal injured tissue (12). Adequate energy delivery is needed to maintain positive energy balance to enable protein utilization for collagen and nitrogen synthesis, rather than its breakdown for energy. When the body is depleted of carbohydrate and fat sources, the liver and kidneys turn to utilizing protein from muscle degradation to metabolize lean body mass into glucose (13). Subsequently, there is less available protein to support the anabolic process of wound healing. Although no published data to date gives specific energy requirements for PI healing in critically ill children, increases in energy and protein provisions have been shown to be beneficial for wound healing in adult populations $(10,14,15)$.

Pre-existing malnutrition, inclusive of obesity, should be taken into consideration when estimating total energy 


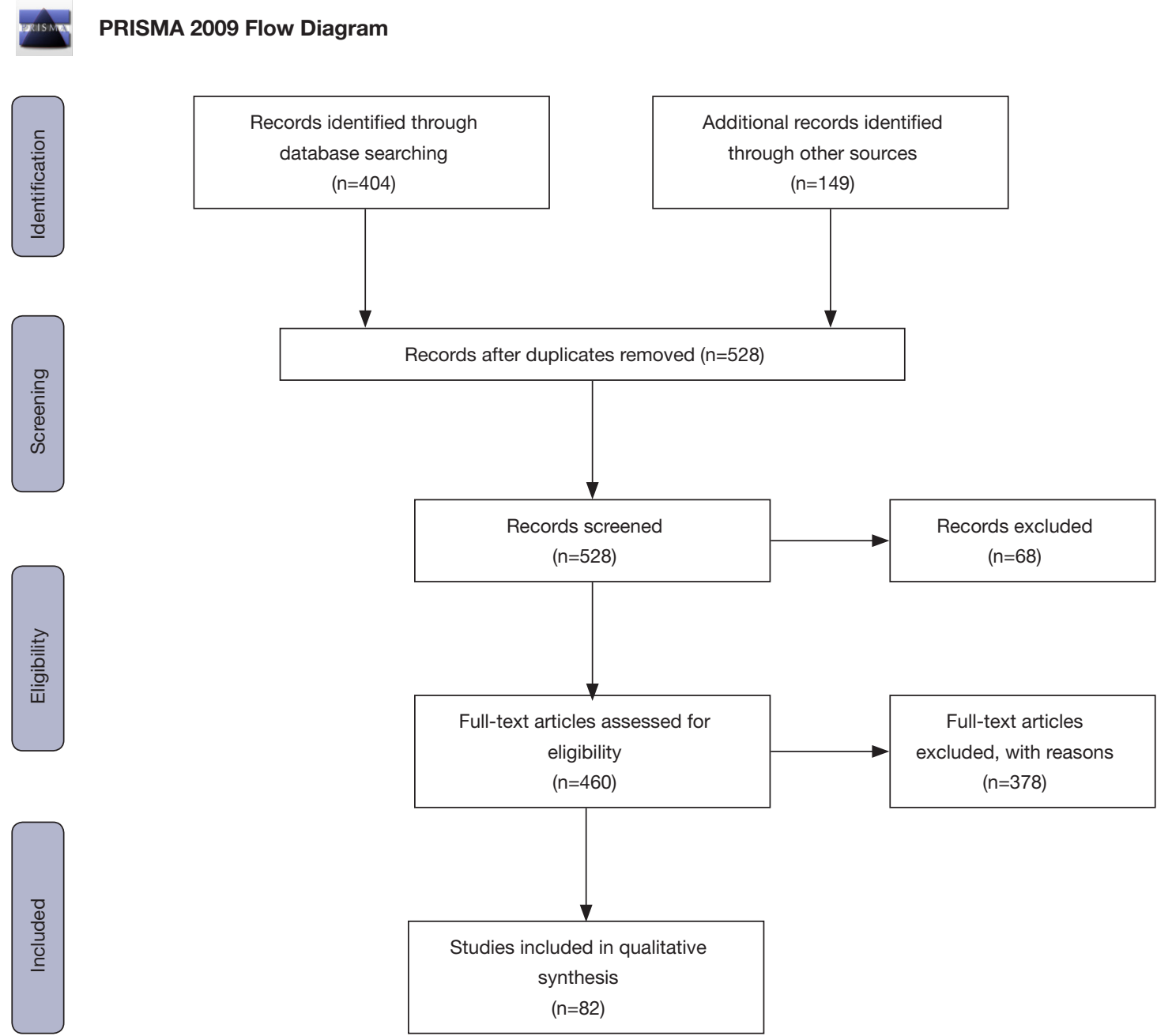

Figure 1 Prisma flow diagram for article screening and inclusion.

needs. Protein-energy malnutrition increases risk for developing PI and impairs wound healing. Children presenting in a malnourished state require higher total energy provision than what is recommended for wellnourished children. In-depth nutrition assessment by a registered dietitian $(\mathrm{RD})$ is an important step to determine energy and protein needs to support catch-up growth and wound healing (16). Ensuring timely and optimal delivery of energy to children with PI is important, however rapid increases in energy delivery should be weighed against the risks of electrolyte imbalances that can occur with refeeding syndrome (17).

\section{Protein}

Protein is needed for maintenance of skin integrity, growth and proliferation of cells, and for fluid and electrolyte balance (18). It has been suggested that the presence of a stage 1-3 PI and/or deep tissue injury (DTI) increases the protein requirement above the U.S. Recommended Daily Allowance (RDA) $(10,19)$. Additionally, the most severe PI (stage 4), have the greatest protein needs for healing (20). Critically ill children in general have increased protein requirements compared to their healthy counterparts, the presence of a PI can further increase these needs. The pediatric critical care nutrition guidelines set forth by the American Society for Parenteral and Enteral Nutrition (ASPEN) and the Society of Critical Care Medicine (SCCM) do not recommend using the RDA for protein as it often underestimates the needs of critically ill children (21). When increasing protein delivery, comorbidities affecting protein metabolism and utilization such as acute kidney 
injury and other organ dysfunction must be taken into consideration.

\section{Fat}

Adequate fat delivery is important to prevent micronutrient deficiencies that can alter skin integrity. Fat is the most concentrated source of energy and can be stored as triglycerides in adipose tissue for later use. Adequate delivery of essential fatty acids (alpha-linolenic and linoleic fatty acids) is important to prevent micronutrient deficiencies that can alter skin integrity (22). Signs of essential fatty acid deficiency include xerosis, seborrheic dermatitis, and poor wound healing (22-24). Fat in the diet is also needed to aid absorption of fat-soluble vitamins A, E, D, and K. Fat should account for a minimum of $10-25 \%$ of overall energy intake, and include essential fats, linolenic and linoleic acid, to avoid deficiency.

\section{Fluid}

Dehydration impairs oxygen delivery to wounded tissue. Stage 1-2 pressure ulcers do not typically necessitate increased fluid requirements above maintenance. However, in adults, fluid needs are increased due to increased insensible losses through wound drainage (10). If a wound drainage device is used, losses from drainage output should also be taken into consideration when assessing fluid status. It is important to manage the fluid needs of critically ill children based on their clinical status as it can be highly variable between patients. It is especially important to prevent worsening fluid overload as patients often receive significant volume resuscitation early in their ICU course that can impact clinical outcomes.

\section{Zinc}

Zinc is an essential mineral that plays a vital role in wound healing, cell division, immune function, and synthesis of DNA and RNA (25). Causality for zinc deficiency can be varied, Corbo et al. (2013) proposed an etiologic classification system of five broad categoriestype I inadequate intake, type II excessive losses, type III malabsorption, and type IV increased demand, and type $\mathrm{V}$ other (26). Zinc deficiency can manifest in numerous ways given its various functions in the body's organ systems. Signs and symptoms of zinc deficiency include, but are not limited to, stunting, altered taste and smell, impaired wound healing, and hypogonadism. Specific cutaneous presentation with zinc deficiency includes skin lesions (typically crusted plaques of perianal and perioral areas), alopecia, and diarrhea (27). Zinc levels used in conjunction with a skin assessment and history help confirm a zinc deficiency. However, zinc levels can be falsely low in times of acute stress and inflammation (26). Therefore, clinical status should be considered when assessing zinc levels, especially in critical illness, and attention should be paid to significant wound drainage or gastrointestinal losses that can increase zinc losses. If zinc deficiency is identified, supplementation with zinc should be initiated. Clinical remission often begins within days of treatment initiation (28). Individuals should not stay on oral zinc supplementation for a prolonged duration as zinc competes with copper absorption. This negatively impacts copper levels which can be counterproductive to wound healing as copper is a necessary cofactor in collagen cross-linking (29). Zinc toxicity is rare, but excessive intake may cause GI distress (30).

Zinc is an important factor in wound healing. Adult studies have shown that zinc supplementation can improve wound healing, but only in patients with deficient levels $(29,31,32)$. Pediatric studies are lacking. Therefore, routine zinc supplementation is not recommended, unless deficiency is observed. Extrapolation of adult studies to the pediatric population is unclear, and more research is needed to better understand and determine zinc supplementation in wound healing particularly in PI in children.

\section{Vitamin C}

Vitamin C is a water-soluble vitamin that functions as an antioxidant, and a cofactor for several enzymes. It plays a key role in fatty acid transport, collagen synthesis, neurotransmitter synthesis, prostaglandin metabolism, and nitric oxide (NO) synthesis (33). Risk factors for deficiency in pediatric patients are developmental delay and autism (due to selective or limited diets), iron overload, periods of oxidative stress, and chemotherapy. Signs of deficiency include poor wound healing, musculoskeletal and joint pain, lethargy, weakness, anorexia, edema, depression, neuropathy, and vasomotor instability. Scurvy is a manifestation of vitamin $\mathrm{C}$ deficiency and can present as perifollicular hemorrhage, petechiae, coiled hair, gingivitis, ecchymosis, mucosal bleeding, and anemia (33). Deficiency from poor intake can occur in as little as three months, though in critically ill children a deficiency may 
occur sooner due to oxidative stress (34). No recommended treatment doses for vitamin $\mathrm{C}$ exist for pediatric patients with wounds or PIs. Vitamin C toxicity is rare, however gastrointestinal upset and oxalate stones have been reported in adults with kidney disease (35).

\section{Vitamin A}

Vitamin A is a fat-soluble vitamin and a potent antioxidant. Vitamin A plays a role in many bodily functions including bone health, gene transcription, cell and tissue differentiation, mucous membrane integrity, collagen synthesis, and immune function (36). With respect to skin integrity and wound healing, vitamin A deficiency can result in impaired healing and increased susceptibility to wound infections. There is no recommended Vitamin A dosing available for wound healing in pediatric patients. Vitamin A should be dosed cautiously in individuals with liver and/ or renal impairment. Acute toxicity in adults with single dosing $>150,000 \mathrm{mcg}$ has been reported to cause nausea, vomiting, headache, vertigo, blurred vision, and increased intracranial pressure (37). Additionally, in infants, a bulging fontanel has been reported in acute and chronic toxicity $(38,39)$. Chronic toxicity in adults who are supplemented with more than 30,000 mcg over an extended period of time can cause irritability, anorexia, skin desquamation, and biochemical liver abnormalities (elevated enzymes, fibrosis, and cirrhosis). Additional abnormal findings observed with vitamin A toxicity in infants include hypercalcemia, hyperphosphatemia, and metastatic calcifications which can occur with intakes of $5,500-6,750 \mathrm{mcg} / \mathrm{d}$ or more for an extended time of 1-3 months (38). Therefore, chronic supplementation with Vitamin A is not typically recommended and should be monitored closely with blood levels.

\section{Iron}

Iron is a mineral that plays an essential role in skin health, mucous membrane integrity, hair, and nails. Iron, along with vitamin $\mathrm{C}$, is needed for collagen synthesis, and is critical for red blood cell production. Clinically, iron deficiency can present as skin pallor, angular cheilitis, koilonychia (spoon nails), fragile nails, pruritus, and brittle hair. Pediatric patients (6-20 months of age) are at risk for iron deficiency anemia, as they transition from iron-fortified formula to cow's milk. Additionally, females are at increased risk for iron deficiency secondary to iron losses with menstruation.
An iron profile is often used to diagnose iron deficiency. However, it should be noted that ferritin, a component of the iron profile, is an acute phase reactant and not reliable in the critically ill patient.

With regards to skin function and integrity, iron deficiency anemia may negatively impact wound strength, but this has not been consistently demonstrated in available human studies (40-42). Thus far, these studies have yet to demonstrate the effects of iron deficiency anemia on the stages of wound healing, with even less available information related to the interplay of iron and PI. There are no current guidelines related to optimum iron dosing for wound healing in pediatric patients. However, if iron deficiency is present, an iron-rich diet and/or iron supplementation is warranted. To enhance absorption, iron should be taken enterally with foods rich in vitamin C.

\section{Arginine}

Arginine is considered a conditionally essential amino acid, which means that in times of acute stress or trauma, the body is unable to produce adequate amounts of arginine to keep up with the physiologic demands (43). Arginine plays a role in collagen synthesis and has immune modulating properties, both of which may contribute to wound healing. Adult studies investigating the use of oral nutrition supplements (ONS) enriched with arginine, zinc, and antioxidants have shown a positive increase in the rate of healing $(14,18,44,45)$. Isolated arginine supplementation is not standard clinical practice, particularly given concerns of its potential to cause excessive nitric oxide $(\mathrm{NO})$ production in critically ill patients with resulting harm (46).

\section{Proposed nutrition management for children with PIs}

Currently, there are no published practice or clinical guidelines for optimum nutrition therapy in pediatric patients with varying stages of PIs. In addition, there are no nutrition risk assessment screening tools validated in critically ill pediatric patients. Thompson et al. addresses nutrition interventions to optimize pediatric wound healing; however, they do not specifically address PIs in critically ill children (47). The clinical pathway developed by Thompson et al. (2014), is to optimize nutrition therapy in pediatric patients with wounds. These authors included wounds that were non-healing surgical incisions, dehisced surgical sites, PIs staged 2-4, wounds requiring wound vac devices, 
and skin breakdown secondary to infections, and nutrition deficiencies (47). Notably, the authors reported increased identification of micronutrient deficiencies (specifically vitamin $\mathrm{C}$ and zinc) as well as improved monitoring of patient's nutritional status in their institution following implementation of the clinical pathway (47). The pathway also allowed for a standardized approach among clinicians to manage nutrition therapy for patients with wounds as identified above.

It is unclear when inadequate nutritional intake begins to negatively affect skin integrity. However, inadequate intake of both macro and micronutrients have a known association with impaired wound healing (10). The increased nutrient needs of pediatric patients to meet the metabolic requirements of age appropriate growth, may be further increased with the presence of PIs, making it imperative to adjust nutrition therapy to support both the basic metabolic requirements and adequate wound healing. This becomes particularly challenging in the hospitalized or critically ill child who often have additional clinical management priorities that potentially compete with nutrition therapy.

Table 2 lists proposed modifications for both macro and micronutrients based on PI staging. Our group developed this proposed table with data modeled from ASPEN's available adult data and is intended for use with children who weigh less than 30 kilograms. The goal of the table is to offer guidance for clinicians to assess, treat, and monitor deficiencies aligned with wound and PI treatment. The tolerable upper limit for vitamin and mineral intake significantly increases from age 4-8 years to that of 9-13 years; therefore, the age of 9 years was used as a cutoff. With no currently available guidelines, the authors chose the $50^{\text {th }}$ percentile for weight in 9-year-old as a point of reference to determine the corresponding weight cut-off limit. A $30 \mathrm{~kg}$ cutoff was determined as this is the weight at approximately the $50^{\text {th }}$ percentile for both 9-year-old boys and girls identified on the Centers for Disease Control (CDC) growth chart for 2- to 20-year-old children.

As with any nutrition intervention, it is imperative to consider the child's medical history and adjust their macro and micronutrient intake based on disease state and the child's nutrition status guided by anthropometric measurements obtained at admission. For example, in patients with cholestatic liver disease, careful choosing of an appropriate multivitamin product is necessary due to malabsorption of fat-soluble vitamins in this population Additionally, Table 3 lists initial doses as well as a subsequent schedule for micro-nutrient monitoring of vitamins and minerals that are essential for adequate wound healing. The table provides proposed lab monitoring frequency and a suggested adjustment of micro-nutrient supplementation based on laboratory measurements. For example, after initiating zinc supplementation at $1 \mathrm{mg} / \mathrm{kg} / \mathrm{day}$ for a low level, if the laboratory value remains deficient on repeat assessment 4 weeks after supplement initiation, the dose should be increased with a plan to reassess at specified intervals. As with all nutrient supplementation, it is important to consider the individual as a whole and take into consideration any underlying organ dysfunction that may interfere with absorption or utilization, while being cognitive of the tolerable upper limits of supplementation for age.

\section{Multidisciplinary team approach}

\section{Role of the clinical nurse}

Routine and thorough skin assessments begin with the patient's nurse, is shared between all members of the clinical team and are crucial in PI prevention and management in pediatric patients. Prevention of PIs starts with a thorough assessment of the patient and their skin integrity upon admission and continues routinely at serial intervals to identify injuries and accurately determine the risk of developing a hospital-acquired PI (53). These evaluations range from detailed physical assessment to review of medical records and include identification and stratification of risk (53). Risk assessments are performed at a frequency based on unit standards, patient diagnosis, acuity, mobility and, other aspects of the patient's condition that may predispose them to a PI (53). If concerns for skin integrity are present, a Skin Champion, a specially trained clinical nurse is consulted to further review the extent of injury. They extend the assessment to include areas where a medical device is in constant contact with the skin. The goal of these assessments is to identify areas that raise concern for development of PI.

\section{Role of the certified wound, ostomy, continence nursing (CWOCN) specialist}

Once a wound or significant skin injury is identified, a CWOCN specialist joins the clinical team for a more specialized skin injury assessment and staging. The CWOCN specialist then makes further recommendations for wound care with the clinical team. Combined with the 


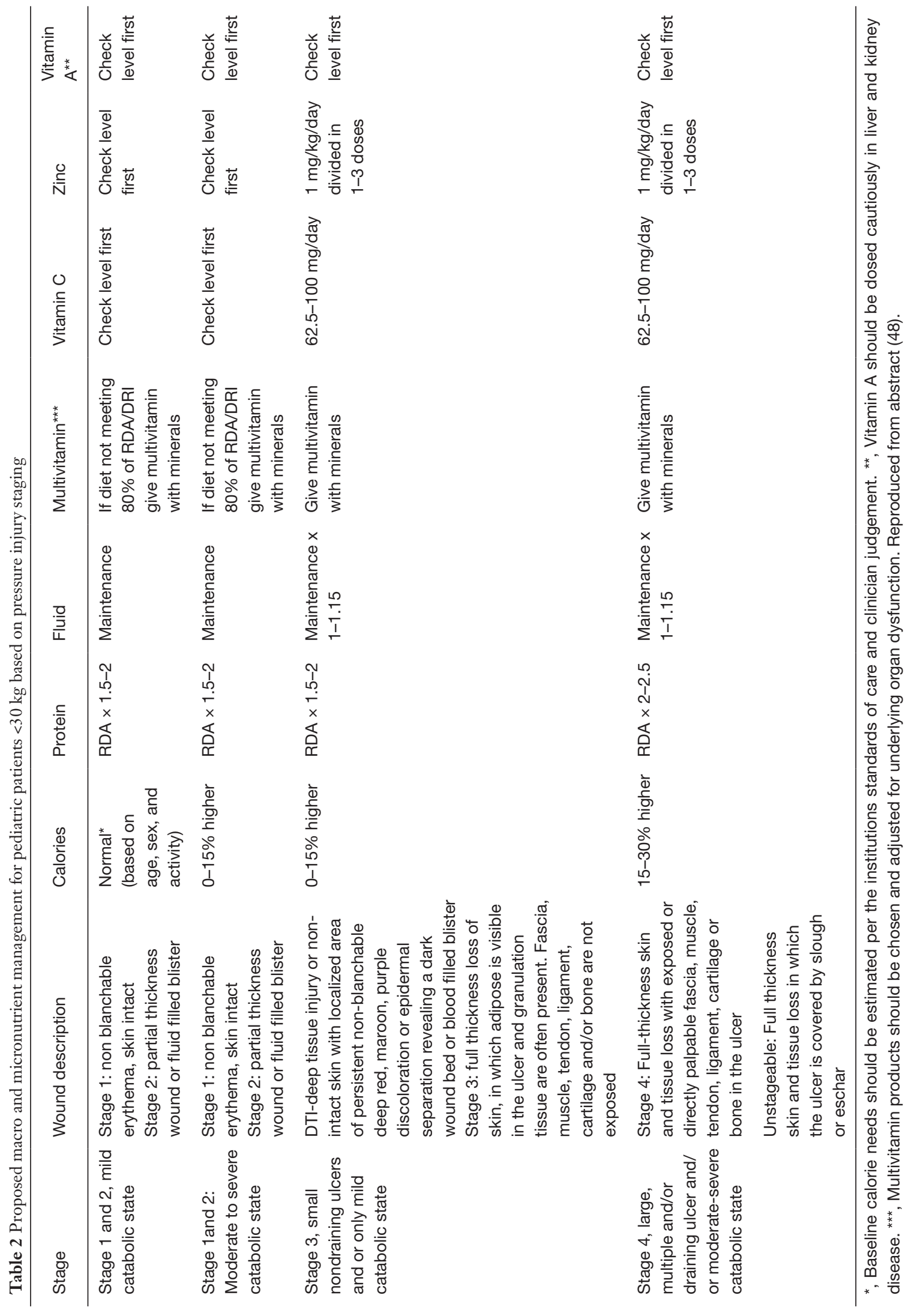




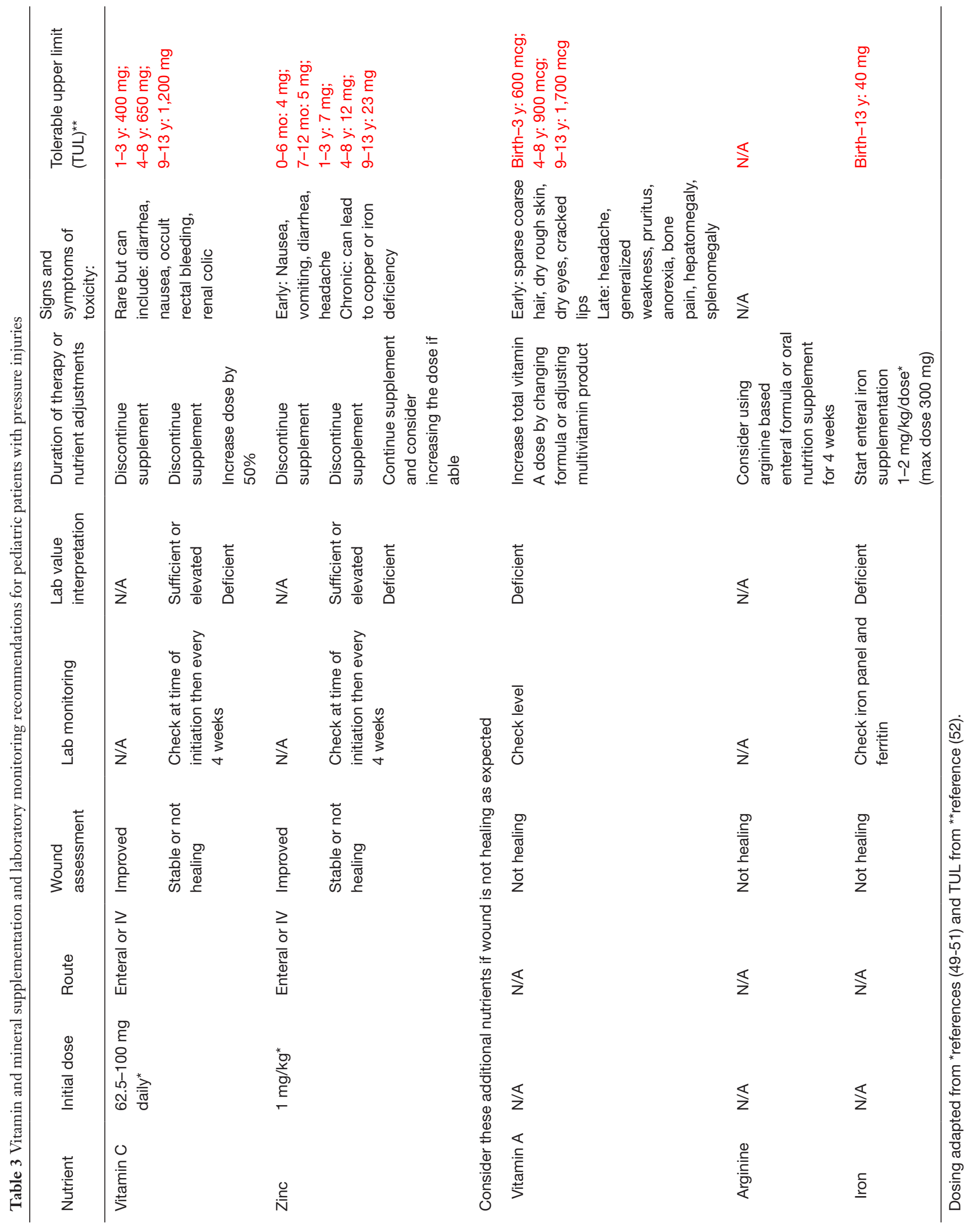




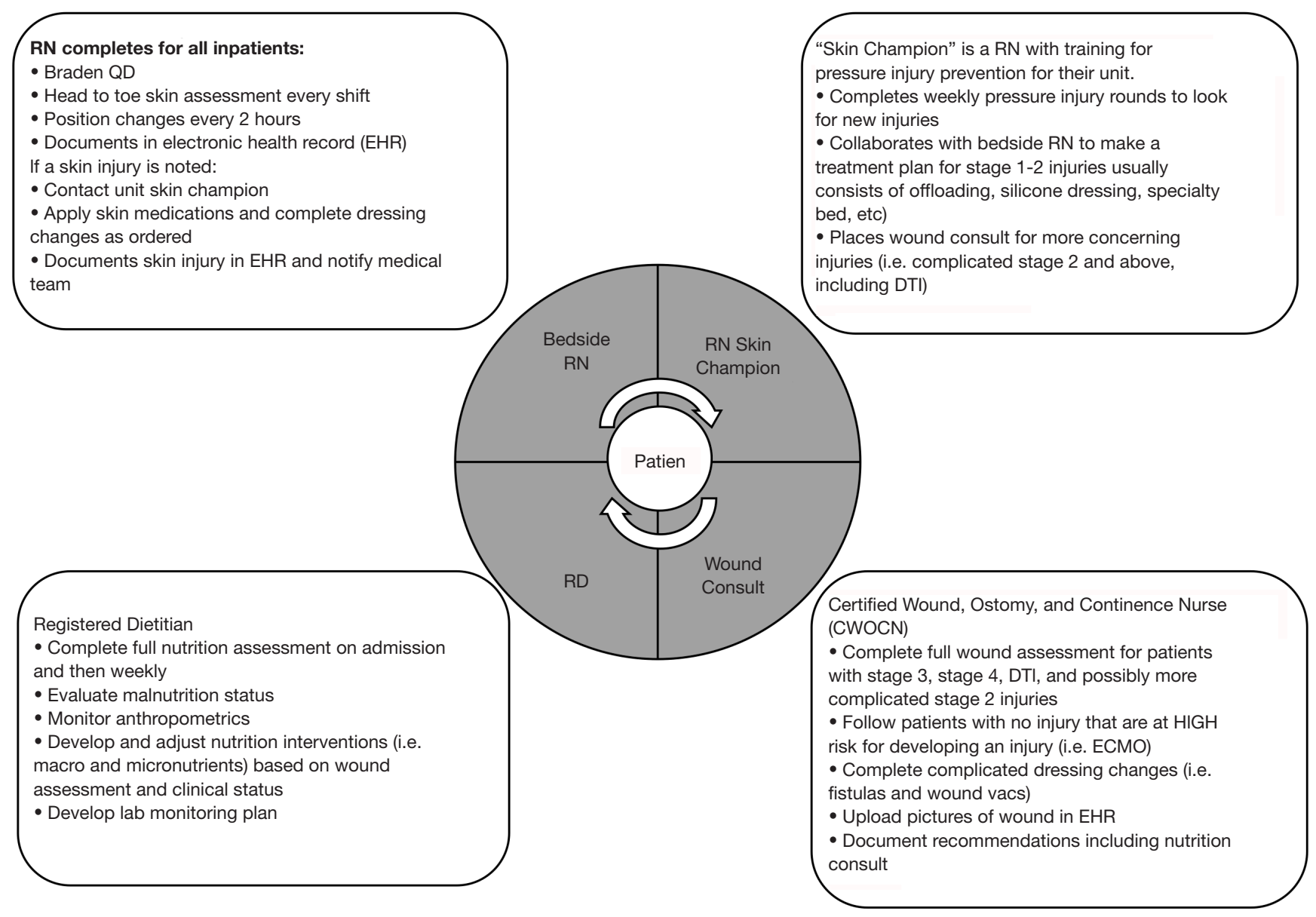

Figure 2 Multidisciplinary team approach to pressure injury prevention and managemen

medical care team, this multidisciplinary approach ensures engagement of the entire team, all focused on keeping the patient and/or family at the forefront of care delivery. Figure 2 illustrates a proposed model for multidisciplinary engagement.

\section{Assessment of patients at risk-Braden $Q D$ scale}

Hospitalized infants and children are at risk for PI, and may possess both intrinsic and extrinsic factors for developing wounds and skin compromise. The child with autoimmune and genetic skin conditions (e.g., pyoderma gangrenosum or epidermolysis bullosa), immunocompromised state (e.g., graft versus host disease, GVHD), requiring surgical intervention, or undergoing therapeutic treatment with potential for skin breakdown (e.g., radiation therapy) have intrinsic factors increasing the risk for PI. Extrinsic factors are additive to the intrinsic factors and increase the risk of the already physiologically compromised child for skin damage or PI that can further complicate their care. Table 4 describes PI with staging.

The Braden Q scale adapted from the Braden Scale (55) is one of the most widely used and is currently the most inclusive scale for measuring risk of PI in the pediatric population (56). In addition to the six original subscales (mobility, activity, sensory perception, moisture, friction and shear, nutrition) from the original Braden Scale, the Braden Q scale adds parameters for tissue perfusion/ oxygenation. The Braden $\mathrm{Q}$ scale has given way to the revised Braden QD scale (5). Tested on a broader age range, from pre-term to 21 years of age, the Braden QD scale also includes patients with cardiac disease and those in a non-critical care environment. Additionally, the Braden QD scale includes numerical scoring to identify the risk of PI from medical devices (47). In hospitalized infants and children, MDRPI is reported to account for up to $71 \%$ of 
Table 4 Pressure injury staging and definitions (54)

\begin{tabular}{ll}
\hline Pressure injury staging & Definitions \\
\hline Stage 1 Pressure Injury & Non-blanchable erythema of intact skin \\
& $\begin{array}{l}\text { Intact skin with a localized area of non-blanchable erythema, which may appear differently in darkly } \\
\text { pigmented skin. Presence of blanchable erythema or changes in sensation, temperature, or firmness may } \\
\text { precede visual changes. Color changes do not include purple or maroon discoloration; these may indicate } \\
\text { deep tissue pressure injury }\end{array}$
\end{tabular}

Stage 2 Pressure Injury

Partial-thickness skin loss with exposed dermis

The wound bed is viable, pink or red, moist, and may present as an intact or ruptured serum-filled blister. Adipose (fat) is not visible and deeper tissues are not visible. Granulation tissue, slough and eschar are not present. These injuries commonly result from adverse microclimate and shear in the skin over the pelvis and shear in the heel. This stage should not be used to describe moisture associated skin damage (MASD) including incontinence-associated dermatitis (IAD), intertriginous dermatitis (ITD), medical adhesive related skin injury (MARSI), or traumatic wounds (skin tears, burns, abrasions)

Stage 3 Pressure Injury

Full-thickness skin loss

Full-thickness loss of skin, in which adipose (fat) is visible in the ulcer and granulation tissue and epibole (rolled wound edges) are often present. Slough and/or eschar may be visible. The depth of tissue damage varies by anatomical location; areas of significant adiposity can develop deep wounds. Undermining and tunneling may occur. Fascia, muscle, tendon, ligament, cartilage and/or bone are not exposed. If slough or eschar obscures the extent of tissue loss this is an Unstageable Pressure Injury

Stage 4 Pressure Injury $\quad$ Full-thickness skin and tissue loss

Full-thickness skin and tissue loss with exposed or directly palpable fascia, muscle, tendon, ligament, cartilage or bone in the ulcer. Slough and/or eschar may be visible. Epibole (rolled edges), undermining and/or tunneling often occur. Depth varies by anatomical location. If slough or eschar obscures the extent of tissue loss this is an Unstageable Pressure Injury.

Unstageable Pressure Injury Obscured full-thickness skin and tissue loss

Full-thickness skin and tissue loss in which the extent of tissue damage within the ulcer cannot be confirmed because it is obscured by slough or eschar. If slough or eschar is removed, a Stage 3 or Stage 4 pressure injury will be revealed. Stable eschar (i.e., dry, adherent, and intact without erythema or fluctuance) on the heel or ischemic limb should not be softened or removed

Deep Tissue Pressure Injury Persistent non-blanchable deep red, maroon or purple discoloration

Intact or non-intact skin with localized area of persistent non-blanchable deep red, maroon, purple discoloration or epidermal separation revealing a dark wound bed or blood filled blister. Pain and temperature change often precede skin color changes. Discoloration may appear differently in darkly pigmented skin. This injury results from intense and/or prolonged pressure and shear forces at the bonemuscle interface. The wound may evolve rapidly to reveal the actual extent of tissue injury, or may resolve without tissue loss. If necrotic tissue, subcutaneous tissue, granulation tissue, fascia, muscle or other underlying structures are visible, this indicates a full thickness pressure injury (Unstageable, Stage 3 or Stage 4). Do not use DTPI to describe vascular, traumatic, neuropathic, or dermatologic conditions

Additional pressure injury definitions

Medical device related pressure injury

Mucosal membrane pressure injury
This describes an etiology. Medical device related pressure injuries result from the use of devices designed and applied for diagnostic or therapeutic purposes. The resultant pressure injury generally conforms to the pattern or shape of the device. The injury should be staged using the staging system

Mucosal membrane pressure injury is found on mucous membranes with a history of a medical device in use at the location of the injury. Due to the anatomy of the tissue, these ulcers cannot be staged 
HAPI (57-60). Identifying PI risk from devices is crucial in the PICU population, where the use of complex medical technology is an integral component of care delivery. The Braden QD scale is validated in the hospitalized pediatric population showing sensitivity of 0.86 (95\% CI: 0.76-0.92) and specificity of 0.59 (95\% CI: $0.55-0.63$ ) (5). It is the only PI risk scale to include medical devices as a potential hazard for occurrence of PI in adult and pediatric populations, and has a nutritional sub-score to determine current nutrition status. The Braden QD scale facilitates the identification of patients at risk for developing PI early in hospitalization allowing for timely delivery of quality, multi-disciplinary patient centered care.

\section{Role of the RD}

Nutrition status at the time of hospitalization is believed to have significant impact on the hospitalized and critically ill pediatric patient and their risk for PI. Patients who present with protein-energy malnutrition on admission are at significant risk for delayed wound healing. Healing requires an optimal nutrition status as wounds lead to a catabolic state which then significantly increase nutritional demands (15). When the body is faced with the added burden of wound healing in the presence of malnutrition it begins to break down lean body mass for protein which then in turn delays the onset of healing. Nutrient deficiencies increase the risk of developing a PI, therefore a nutritional plan inclusive of necessary supplementation for at risk patients should be executed under the direction of a $\mathrm{RD}(61)$.

The RD is uniquely qualified in many ways to serve as an integral member of the clinical care team. The expertise of a RD to perform a nutritional assessment within the initial 48 hours of PICU admission can help identify nutritional pitfalls, sub-optimal nutrition status, and patients at increased risk for PI early in the hospital stay (61). Nutrition assessment includes determining the patient's needs and goals for macronutrients and micronutrient supplementation specific to wound healing. These assessments should then continue to be performed weekly after admission to re-evaluate the patient throughout the course of their PICU stay (61). Following a thorough assessment, the $\mathrm{RD}$ can recommend an appropriate oral, enteral, parenteral or combined regimen to meet the patient's identified nutritional goals.

Working with the family, incorporating home food preferences (where appropriate), the $\mathrm{RD}$ is integral in keeping the patient and family involved, and in the forefront while devising a nutrition therapy plan of care appropriate for the patient's clinical illness status and the mode by which the nutritional plan will be delivered. Working with the clinical staff, the $\mathrm{RD}$ will incorporate potential interruptions in nutrient delivery to create a reliable and achievable feeding plan for use in the hospital and potentially in the home setting. Diet modification is directed by the RD as necessary to accommodate for food allergies, cultural dietary customs, dietary preferences such as vegetarianism, and health conditions such as diabetes, obesity or kidney disease that require a specific therapeutic diet to optimize established guidelines necessary for wound healing. In addition, the clinical RD is knowledgeable about the availability of protein and/or calorie modular supplements commercially available, or able to be created with household ingredients per patient and family preference. In addition, the RD can assess vitamin and mineral laboratory measurement data and recommend supplementation and monitoring where appropriate to enhance wound healing.

If the oral route is not sufficient to meet the patient's increased energy and protein needs prescribed by the RD, supplemental tube feedings may then be recommended. This can be provided via an enteral feeding tube. The patient's family can also be advised (by the clinical team) to consider semi-permanent enteral access such as a percutaneous gastrostomy (PEG) tube to ensure adequate nutrition intake if oral intake cannot be maintained to support nutritional needs, and skin integrity. Development of multiple or chronic pressure injuries increase risk of infection, arthritis and osteomyelitis, leading to sepsis and possibly death. RDs possess the knowledge and skills to translate the recent research into clinical practice.

\section{Limitations}

There are limitations to this work to be acknowledged. There is a significant void in the literature giving guidance for optimal nutrition therapy for pediatric patients with or at risk for PI wound care of device induced skin injury. The scoping review performed was limited to recent literature in the last ten years, however, lacks definitive evidence or guidelines to inform nutrition therapy or supplementation specific to this population of patients. While the recommendations given are useful to our institution, a large quaternary-care, urban, academic children's hospital, they may not be the same resources available in other institutions. Future work should entail close monitoring of nutrition therapy, micronutrient supplementation and 
laboratory monitoring to establish efficacy and determine impact on patient outcomes. Research and review are urgently needed.

\section{Conclusions}

There is a substantial gap in knowledge and research in how nutrition therapy influences PI healing in pediatric patients. Hospitalized children, especially critically ill children, are at increased risk of PI development. Suboptimal nutrition status can impair, and delay wound healing, and attention should be paid to macro and micronutrient intake. Nutrition status plays a critical role in wound healing, and children have unique needs given their increased metabolic demands compared to adults. These proposed initial recommendations for assessing children with PI are meant to ensure adequate nutritional therapy to optimize wound healing. However, research to further build upon these recommendations is necessary to better define the role of nutrition therapy and supplementation in PI prevention and healing among hospitalized children.

\section{Acknowledgments}

Funding: None.

\section{Footnote}

Provenance and Peer Review: This article was commissioned by the editorial office, Translational Pediatrics for the series "Pediatric Critical Care". The article has undergone external peer review.

Peer Review File: Available at https://dx.doi.org/10.21037/ tp-21-3

Conflicts of Interest: All authors have completed the ICMJE uniform disclosure form (available at https://dx.doi. org/10.21037/tp-21-3). The series "Pediatric Critical Care" was commissioned by the editorial office without any funding or sponsorship. VS served as the unpaid Guest Editor of the series and serves as an unpaid editorial board member of Translational Pediatrics from Oct 2019 to Sep 2021. Dr. SYI reports receiving reduced fee as conference faculty from ASPEN and SCCM, outside the submitted work. The other authors have no conflicts of interest to declare.
Ethical Statement: The authors are accountable for all aspects of the work in ensuring that questions related to the accuracy or integrity of any part of the work are appropriately investigated and resolved.

Open Access Statement: This is an Open Access article distributed in accordance with the Creative Commons Attribution-NonCommercial-NoDerivs 4.0 International License (CC BY-NC-ND 4.0), which permits the noncommercial replication and distribution of the article with the strict proviso that no changes or edits are made and the original work is properly cited (including links to both the formal publication through the relevant DOI and the license). See: https://creativecommons.org/licenses/by-nc-nd/4.0/.

\section{References}

1. Razmus I, Bergquist-Beringer S. Pressure Injury Prevalence and the Rate of Hospital-Acquired Pressure Injury Among Pediatric Patients in Acute Care. J Wound Ostomy Continence Nurs 2017;44:110-7.

2. Schindler CA, Mikhailov TA, Cashin SE, Malin S, Christensen M, Winters JM. Under pressure: preventing pressure ulcers in critically ill infants. J Spec Pediatr Nurs 2013;18:329-41.

3. Visscher M, King A, Nie AM, et al. A quality-improvement collaborative project to reduce pressure ulcers in PICUs. Pediatrics 2013;131:e1950-60.

4. Stellar, JJ. Hospital-acquired skin injury: Nurse led interventions to improve outcomes. In: Scientific and Clinical Abstracts From the WOCN(R) Society's 45th Annual Conference. Seattle, Washington, USA; 2013.

5. Curley MAQ, Hasbani NR, Quigley SM, et al. Predicting Pressure Injury Risk in Pediatric Patients: The Braden QD Scale. J Pediatr 2018;192:189-195.e2.

6. Kulik LA, Hasbani NR, Stellar JJ, et al. Hospital-Acquired Pressure Injuries in Children With Congenital Heart Disease: Prevalence and Associated Factors. Pediatr Crit Care Med 2019;20:1048-56.

7. Kottner J, Wilborn D, Dassen T. Frequency of pressure ulcers in the paediatric population: a literature review and new empirical data. Int J Nurs Stud 2010;47:1330-40.

8. Schlüer AB, Schols JM, Halfens RJ. Risk and associated factors of pressure ulcers in hospitalized children over 1 year of age. J Spec Pediatr Nurs 2014;19:80-9.

9. Baharestani MM, Ratliff CR. Pressure ulcers in neonates and children: an NPUAP white paper. Adv Skin Wound 
Care 2007;20:208, 210, 212, 214, 216, 218-20.

10. Munoz N, Posthauer ME, Cereda E, et al. The Role of Nutrition for Pressure Injury Prevention and Healing: The 2019 International Clinical Practice Guideline Recommendations. Adv Skin Wound Care 2020;33:123-36.

11. Peters MDJ, Marnie C, Tricco AC, et al. Updated methodological guidance for the conduct of scoping reviews. JBI Evid Synth 2020;18:2119-26.

12. Molnar JA, Underdown MJ, Clark WA. Nutrition and Chronic Wounds. Adv Wound Care (New Rochelle) 2014;3:663-81.

13. Tappenden K. Intake: digestion, absorption, transportation, and excretion of nutrients. In: Krause's Food \& the Nutrition Care Process. 14th edition. St. Louis, Missouri: Elsevier, 2017:2-16.

14. Cereda E, Klersy C, Rondanelli M, et al. Energy balance in patients with pressure ulcers: a systematic review and meta-analysis of observational studies. J Am Diet Assoc 2011;111:1868-76.

15. Demling RH. Nutrition, anabolism, and the wound healing process: an overview. Eplasty 2009;9:e9.

16. Becker P, Carney LN, Corkins MR, et al. Consensus statement of the Academy of Nutrition and Dietetics/ American Society for Parenteral and Enteral Nutrition: indicators recommended for the identification and documentation of pediatric malnutrition (undernutrition). Nutr Clin Pract 2015;30:147-61.

17. Pulcini CD, Zettle S, Srinath A. Refeeding Syndrome. Pediatr Rev 2016;37:516-23.

18. Breslow RA, Hallfrisch J, Guy DG, et al. The importance of dietary protein in healing pressure ulcers. J Am Geriatr Soc 1993;41:357-62.

19. Nieman Carney L, Blair J. Assessment of Nutrition Status by Age and Determining Nutrient Needs. In: The ASPEN Pediatric Nutrition Support Core Curriculum. Maryland: American Society for Parenteral and Enteral Nutrition; 2010:409-13.

20. Lee SK, Posthauer ME, Dorner B, et al. Pressure ulcer healing with a concentrated, fortified, collagen protein hydrolysate supplement: a randomized controlled trial. Adv Skin Wound Care 2006;19:92-6.

21. Mehta NM, Skillman HE, Irving SY, et al. Guidelines for the Provision and Assessment of Nutrition Support Therapy in the Pediatric Critically Ill Patient: Society of Critical Care Medicine and American Society for Parenteral and Enteral Nutrition. JPEN J Parenter Enteral Nutr 2017;41:706-42.
22. Jeppesen PB, Christensen MS, Høy CE, et al. Essential fatty acid deficiency in patients with severe fat malabsorption. Am J Clin Nutr 1997;65:837-43.

23. Piper CM, Carroll PB, Dunn FL. Diet-induced essential fatty acid deficiency in ambulatory patient with type I diabetes mellitus. Diabetes Care 1986;9:291-3.

24. Mordarski B, Wolff J. Nutrition Focused Physical Exam Pocket Guide. Chicago: Academy of Nutrition and Dietetics; 2015.

25. Tuerk MJ, Fazel N. Zinc deficiency. Curr Opin Gastroenterol 2009;25:136-43.

26. Corbo MD, Lam J. Zinc deficiency and its management in the pediatric population: a literature review and proposed etiologic classification. J Am Acad Dermatol 2013;69:616624.e1.

27. Lekwuttikarn R, Teng JMC. Cutaneous manifestations of nutritional deficiency. Curr Opin Pediatr 2018;30:505-13.

28. Jen M, Yan AC. Syndromes associated with nutritional deficiency and excess. Clin Dermatol 2010;28:669-85.

29. Williams JZ, Barbul A. Nutrition and wound healing. Surg Clin North Am 2003;83:571-96.

30. Yanagisawa H. Zinc deficiency and clinical practice. JMAJ 2004;47:359-64.

31. Ayello EA, Thomas DR, Litchford MA. Nutritional aspects of wound healing. Home Healthc Nurse. 1999;17(11):719-29; quiz 730.

32. Scholl D, Langkamp-Henken B. Nutrient recommendations for wound healing. J Intraven Nurs 2001;24:124-32.

33. Winston K, Christie J., Saba M. et al. Water-Soluble Essential Micronutrients. Maryland. In: ASPEN Pediatric Nutrition Support Core Curriculum. Maryland: American Society for Parenteral and Enteral Nutrition; 2010:56-73.

34. Dao DT, Anez-Bustillos L, Cho BS, et al. Assessment of Micronutrient Status in Critically Ill Children: Challenges and Opportunities. Nutrients 2017;9:1185.

35. Fukushima R, Yamazaki E. Vitamin C requirement in surgical patients. Curr Opin Clin Nutr Metab Care 2010;13:669-76.

36. Zinder R, Cooley R, Vlad LG, et al. Vitamin A and Wound Healing. Nutr Clin Pract 2019;34:839-49.

37. Bendich A, Langseth L. Safety of vitamin A. Am J Clin Nutr 1989;49:358-71.

38. Persson B, Tunell R, Ekengren K. Chronic Vitamin A Intoxication During the First Half Year of Life; Description Of 5 Cases. Acta Paediatr Scand 1965;54:49-60.

39. Olson JA. Adverse effects of large doses of vitamin A and 
retinoids. Semin Oncol 1983;10:290-3.

40. Jonsson K, Jensen JA, Goodson WH 3rd, et al. Tissue oxygenation, anemia, and perfusion in relation to wound healing in surgical patients. Ann Surg 1991;214:605-13.

41. Pavlidis TE, Galatianos IN, Papaziogas BT, et al. Complete dehiscence of the abdominal wound and incriminating factors. Eur J Surg 2001;167:351-4; discussion 355 .

42. Agarwal V, Sachdev A, Singh R, et al. Autoimmune hemolytic anemia associated with benign ovarian cyst: a case report and review of literature. Indian J Med Sci 2003;57:504-6.

43. Zhou M, Martindale RG. Arginine in the critical care setting. J Nutr 2007;137:1687S-92S.

44. Banks MD, Ross LJ, Webster J, et al. Pressure ulcer healing with an intensive nutrition intervention in an acute setting: a pilot randomised controlled trial. J Wound Care 2016;25:384-92.

45. Neyens JCL, Cereda E, Meijer EP, et al. Arginineenriched oral nutritional supplementation in the treatment of pressure ulcers: A literature review. Wound Med 2017;16:46-51.

46. Thompson C, Fuhrman MP. Nutrients and wound healing: still searching for the magic bullet. Nutr Clin Pract 2005;20:331-47.

47. Thompson KL, Leu MG, Drummond KL, et al. Nutrition Interventions to Optimize Pediatric Wound Healing: An Evidence-Based Clinical Pathway. Nutr Clin Pract 2014;29:473-82.

48. ASPEN NUTRITION SCIENCE \& PRACTICE CONFERENCE: March 28-31, 2020, Tampa, Florida: Vars Candidates, Trainee Awards, Best of ASPEN (Topic Awards), International Awards, Abstracts of Distinction, Posters of Distinction and Other Abstracts. JPEN J Parenter Enteral Nutr 2020;44:382. Erratum in: JPEN J Parenter Enteral Nutr 2021;45:215.

49. Multivitamin products. In: Lexi-drugs online [database on the Internet]. Hudson (OH): Lexicomp Inc.: 2021 [updated 14 Jul 2021; cited 18 Jul 2021]. Available online: http:// online.lexi.com

50. Ascorbic acid. In: Lexi-drugs online [database on the Internet]. Hudson (OH): Lexicomp Inc.: 2021 [updated 13 May 2021; cited 18 Jul 2021]. Available online: http:// online.lexi.com

51. Zinc gluconate. In: Lexi-drugs online [database on the Internet]. Hudson (OH): Lexicomp Inc.: 2021 [updated 29 Jan 2021; cited 18 Jul 2021]. Available online: http:// online.lexi.com

52. Dietary Reference Intakes: Applications in Dietary Assessment [Internet]. Washington, D.C.: National Academies Press; 2000 [cited 2020 Dec 27]. Available online: http://www.nap.edu/catalog/9956

53. Chamblee TB, Pasek TA, Caillouette CN, et al. CE: How to Predict Pediatric Pressure Injury Risk with the Braden QD Scale. Am J Nurs 2018;118:34-43.

54. Edsberg LE, Black JM, Goldberg M, et al. Revised National Pressure Ulcer Advisory Panel Pressure Injury Staging System: Revised Pressure Injury Staging System. J Wound Ostomy Continence Nurs 2016;43:585-97.

55. Braden B, Bergstrom N. A conceptual schema for the study of the etiology of pressure sores. Rehabil Nurs 1987;12:8-12.

56. Curley MA, Razmus IS, Roberts KE, et al. Predicting pressure ulcer risk in pediatric patients: the Braden $\mathrm{Q}$ Scale. Nurs Res 2003;52:22-33.

57. Stellar J. Hospital-acquired pressure injury in pediatrics: patterns and characteristics of injuries over a 6-year period. In: Scientific and Clinical Abstracts From the WOCN® Society's 49th Annual Conference: Salt Lake City, Utah May 19-23, 2017 [Internet]. 2017 [cited 2020 Nov 20]. p. S1-72. Available online: http://journals.lww. com/00152192-201705001-00001

58. Miske LJ, Stetzer M, Garcia M, et al. Airways and Injuries: Protecting Our Pediatric Patients from Respiratory Device-Related Pressure Injuries. Crit Care Nurs Clin North Am 2017;29:187-204.

59. Murray JS, Noonan C, Quigley S, et al. Medical devicerelated hospital-acquired pressure ulcers in children: an integrative review. J Pediatr Nurs 2013;28:585-95.

60. Boyar V. Outcomes of a Quality Improvement Program to Reduce Hospital-acquired Pressure Ulcers in Pediatric Patients. Ostomy Wound Manage 2018;64:22-8.

61. Boylan C. Paediatric pressure injuries: considerations for this patient cohort. Wound Practice and Research 2020;28:84-9.

Cite this article as: Berry KG, Seiple SM, Stellar JJ, Nagle ML, Curry K, Immel A, James R, Srinivasan V, Mascarenhas MR, Garrett A, Irving SY. A scoping review to inform a multi-disciplinary approach for nutrition therapy in critically ill children with pressure injuries. Transl Pediatr 2021;10(10):2799-2813. doi: 10.21037/tp-21-3 